\title{
PREDICTION OF THE LONG-TERM METABOLIC SUCCESS OF THE PANCREATIC GRAFT FUNCTION
}

\author{
Alberto Battezzati,,${ }^{1,3,4}$ Stefano Benedini,${ }^{3}$ Rossana Caldara,${ }^{1}$ Giliola Calori,${ }^{1}$ \\ Antonio Secchi, ${ }^{3}$ Guido Pozza, ${ }^{2}$ And Livio Luzi ${ }^{3}$
}

Department of Internal Medicine, San Raffaele Scientific Institute; San Raffaele University; and University of Milan; Milan, Italy

\begin{abstract}
Background. Strategies to prevent the return to the diabetic state for graft loss or failure or any other cause after pancreas transplantation require the identification of the subjects at risk. This study evaluated whether daily glucose, insulin, and c-peptide profiles and studies of insulin sensitivity and secretion after transplantation predict pancreatic graft failure.

Methods. Fifty-three subjects with type 1 diabetes with end-stage renal failure who received a combined pancreas and kidney transplant underwent the following procedures 1 year after transplantation: 1-day metabolic profiles, sampling every 2 hours for plasma glucose, serum insulin, and c-peptide $(n=51)$; an intravenous glucose tolerance test (IVGTT) to evaluate insulin secretion $(n=48)$; and an euglycemic insulin clamp to evaluate insulin sensitivity ( $M$ value, $n=14)$. The recipients were then followed up to 8 years (mean follow-up $4.8 \pm 0.3$ years) to evaluate the return to the diabetic state.
\end{abstract}

Results. Survival analysis showed that plasma glucose in the profiles and insulin secretion in IVGTT were strongly related to the risk of returning to the diabetic state. A cutoff value of mean daily plasma glucose $>127 \mathrm{mg} / \mathrm{dL}$, corresponding to the top quartile of the mean plasma glucose distribution in the profiles, predicted the return to the diabetic state within 4 years from transplantation with a 93\% specificity and a $100 \%$ sensitivity. A cutoff value of insulin delta peak $<32 \mu \mathrm{U} / \mathrm{ml}$ in the IVGTT predicted the return to the diabetic state within 4 years from transplantation with a $\mathbf{7 5 \%}$ specificity and a $\mathbf{7 5 \%}$ sensitivity. In contrast, the $M$ value in the clamp was devoid of predictive value.

Conclusions. This study indicates that the mean 24-h plasma glucose 1 year after transplantation is the strongest predictor of the return to the diabetic state. The risk is related to defects in insulin secretion and not to insulin resistance. Metabolic profiles can be used to screen the subjects at risk to strictly monitor the graft function and to investigate early determinants of graft failure.

The return to the diabetic state for graft loss or failure or any other cause endangers a significant subset of pancreatic graft recipients (1), but the identification of the subjects at

\footnotetext{
${ }^{1}$ Department of Internal Medicine, San Raffaele Scientific Institute.

${ }^{2}$ San Raffaele University, Milan, Italy.

${ }^{3}$ University of Milan, Milan, Italy.

${ }^{4}$ Address correspondence to: Alberto Battezzati, MD, Amino Acid and Stable Isotopes Laboratory, San Raffaele Scientific Institute, Via Olgettina, 60, 20132 Milano, Italy. E-mail: battezzati. alberto@hsr.it.
}

risk is difficult and often delayed so that an appropriate treatment is not feasible. The available biochemical parameters and imaging tools (2-7) lack diagnostic accuracy to detect early the failure of the transplanted pancreas. Reasons for pancreas failure in the long term may relate to the donor, to the surgical manipulation of the graft, or to the recipient. Several factors that potentially affect graft survival have been identified, including HLA and ABO mismatching and the surgical procedure (8). In addition, some characteristics of the recipients, such as the predisposition to type 2 diabetes, to infections, and to the recurrence of $\beta$-cell autoimmunity, may affect graft survival. Thus, it is possible to hypothesize that the duration of graft survival is in part already determined at the time of transplantation.

When investigating the frequency and the pathogenesis of hypoglycemia after pancreas transplantation (9), we previously noticed that hypoglycemic values recorded in 24 -h metabolic profiles predicted a better metabolic function in the following years. On the basis of these observations, we retrospectively explored whether biochemical parameters derived from metabolic studies had a predictive value for the long-term survival of the pancreatic graft function. Because insulin resistance and altered insulin secretion play a major role in deranging glucose tolerance, we also measured the predictive value of insulin sensitivity and insulin secretion evaluated by means of the euglycemic hyperinsulinemic clamp and the intravenous glucose tolerance test (IVGTT) performed after pancreas transplantation.

\section{METHODS}

Subjects. Fifty-three patients with type 1 diabetes with end-stage uremia, who received a combined pancreas and kidney transplant from a cadaveric donor between 1985 and 1995, were included in this analysis. In all subjects, the pancreas was anastomosed to the iliac vessels. When a whole pancreas was transplanted $(n=37)$, the exocrine pancreatic juice was drained into the bladder (10); when a segmental pancreas was transplanted $(n=16)$, the pancreatic duct was injected with neoprene (11). The characteristics of the subjects are reported in Table 1 . At the time of the study, 1 year after transplantation, all patients were on a triple immunosuppressive regimen; the mean doses are outlined in Table 1 . Specifically, the dose of cyclosporine was adjusted to fit into concentration ranges that changed over time, depending on the assay that was used. From 1985 to 1990 (patients 1-17), the range was 400 to $1200 \mathrm{ng} / \mathrm{ml}$ for plasma concentrations measured with polyclonal antibodies; from 1990 to 1994 (patients 18-43), the range was 300 to $600 \mathrm{ng} / \mathrm{ml}$ for whole blood concentrations measured with polyclonal antibodies; and from 1994 (patients 44-53), the range was 100 to $250 \mathrm{ng} / \mathrm{ml}$ for whole blood concentrations measured with monoclonal antibodies.

Study protocol. One year after the transplant, the pancreas recipients who were insulin independent were admitted to the hospital 
TABLE 1. Clinical characteristics of the pancreas recipients 1 year after transplantation

\begin{tabular}{lc}
\hline Sex $(\mathrm{m} / \mathrm{f})$ & $30 / 23$ \\
Duration of diabetes (yr) & $25.5 \pm 0.9$ \\
Age at transplant $(\mathrm{yr})$ & $38.2 \pm 1.1$ \\
Segmentary/total & $16 / 37$ \\
Cold ischemia time (min) & $557 \pm 42$ \\
Weight $(\mathrm{kg})$ & $62.6 \pm 1.4$ \\
Prednisone (mg/day) & $10.1 \pm 0.2$ \\
Cyclosporine (mg/day) & $335 \pm 14$ \\
Azathioprine (md/day) & $64 \pm 4$ \\
Creatinine (mg/dL) & $1.3 \pm 0.1$ \\
\hline
\end{tabular}

for a metabolic assessment that consisted of the following procedures: 24 -h metabolic profile $(n=51)$ that consisted of sampling every 2 hours plasma glucose, serum free-insulin, and c-peptide concentrations. During the profiles, the subjects received an isocaloric diet fractionated in a breakfast, lunch, and dinner of their choice.

IVGTT. Forty-eight subjects underwent an IVGTT as previously described $(0.3 \mathrm{~g} / \mathrm{kg}$ of dextrose administered as an intravenous bolus followed by a rapid sampling of serum insulin concentrations), and the peak incremental insulin response was used as an index of insulin secretion.

Euglycemic hyperinsulinemic clamp $(n=14)$. Briefly, a prime-continuous infusion of crystalline human insulin (Actrapid HM, Novo Nordisk, Copenhagen, Denmark) was administered at the rate of 1 $\mathrm{mU} \cdot \mathrm{kg}^{-1} \cdot \mathrm{min}^{-1}$ for 2 hours to achieve and maintain an increment in plasma insulin concentrations of approximately $430 \mathrm{pmol} / \mathrm{L}$. The plasma glucose concentration was maintained at the basal concentration by determining it at 5-min intervals with a Beckman glucose analyzer (Beckman Instruments, Fullerton, CA) and by periodic adjustment of a $20 \%$ glucose infusion on the basis of a negative feedback principle. The $\mathrm{M}$ value was calculated from the rate of glucose infusion necessary to keep euglycemia in the second hour of the clamp, corrected for changes in glucose concentration and urinary glucose losses (12). To correctly estimate the whole-body glucose disposal during hyperinsulinemia, the calculation of the $\mathrm{M}$ value assumes that the endogenous glucose production is completely suppressed. This assumption was previously tested in a subset of the recipients with the aid of glucose tracers, and the results in these subjects have been published (13).

Analytical methods. The aliquots of blood for the measurement of glucose were placed into tubes that contained lithium heparin and lithium iodoacetate and immediately centrifuged, then the plasma was decanted and refrigerated at $4^{\circ} \mathrm{C}$ until the assay with the hexokinase method (Boehringer Mannheim, Germany) (14). The blood aliquots for free-insulin and c-peptide were prepared as previously described and were measured by standard RIA methods (15).

Follow-up. Table 2 shows the individual durations of the followup. The return to the diabetic state was defined by fasting plasma glucose $>140 \mathrm{mg} / \mathrm{dL}$ coupled with $\mathrm{HbA}_{\mathrm{c}}>6.5 \%$ and/or initiation of insulin therapy. Six subjects dropped out for reasons independent from the pancreas (cerebral bleeding, neoplasm, or abdominal surgery). Fourteen patients experienced the return to the diabetic state. The failure of the pancreatic function was definitive in 13 of these subjects, and only transient in one patient. In the third year, this recipient (\#31) had an episode of acute uveitis that was treated with an increased dose of steroids. He also underwent surgery for bilateral cataracts immediately before the metabolic tests. He recovered in a few months and had a satisfactory pancreatic function in the following 2 years. This subject was defined free of diabetes at the end of the observation period.

Statistical analysis. The daily mean, minimum, maximum, and the maximum excursion on the day of the profile were calculated for plasma glucose, serum free-insulin, and serum c-peptide concentrations. The contribution to the risk of returning to the diabetic state of these variables in the profiles of the $M$ value in the insulin clamp and of the incremental insulin response in the IVGTT was evaluated by Cox proportional hazards regression analysis. Kaplan-Meier survival curves were used to show the survival of the pancreatic function in the recipients. Analysis with receiver operating characteristic (ROC) methodology was used to evaluate the contribution of mean daily glucose in the profiles and of the insulin response in the IVGTT to predict pancreatic failure at 4 years.

\section{RESULTS}

The subjects were followed for $4.8 \pm 0.3$ years. The individual data in the profiles, in the IVGTT, and in the clamp are reported in Table 2 with the duration and the outcome of the follow-up.

24-h metabolic profiles. The variables derived from plasma glucose were significantly associated with the risk of returning to the diabetic state (daily mean, $P=0.0004$; minimum, $P=0.0007$; maximum, $P=0.0005$; and excursion, $P=0.0012$ ). The mean glucose had the greatest association with the hazard function: an increment of 1 standard deviation $(30 \mathrm{mg} / \mathrm{dL})$ in the mean glucose increased the risk of returning to the diabetic state 1.645 (range, 1.25 to 2.17 ) times. The risk increased briskly in the top quartile of the distribution of the mean glucose concentrations, corresponding to a cutoff value of $127 \mathrm{mg} / \mathrm{dL}$. The top panel of Figure 1 shows the Kaplan-Meier survival curve of pancreatic function based on this cutoff. The mean survival time in the subjects whose mean daily glucose was $\leq 127 \mathrm{mg} / \mathrm{dL}$ was $7.52 \pm 0.37$ years, whereas in the subjects whose mean daily glucose was $>127 \mathrm{mg} / \mathrm{dL}$, the mean survival was $3.07 \pm 0.30$ years $(P<0.00001$ with log-rank test). The bottom panel of Figure 1 shows the relationship between survival of the pancreatic function at 4 years and mean daily glucose in the subjects who were transplanted at least 4 years previously. ROC analysis showed that the cutoff value of $127 \mathrm{mg} / \mathrm{dL}$ predicted a 4 -year survival with a $93 \%$ specificity and a $100 \%$ sensitivity. In contrast to glucose, the variables derived from insulin and c-peptide concentrations were not associated to the hazard function.

Insulin clamp. The $\mathrm{M}$ value calculated during the euglycemic clamp was not associated with the risk of returning to the diabetic state. The top panel of Figure 2 shows that there was no difference in the function survival of the subjects with the $\mathrm{M}$ value in the lowest quartile compared to the others. The bottom panel of the figure shows that there was no relationship between survival of the pancreatic function at 4 years and $\mathrm{M}$ value in the subjects who were transplanted at least 4 years previously.

IVGTT. The peak incremental response in insulin concentration was significantly associated to the risk of returning to the diabetic state $(P<0.001)$. The top panel of Figure $3 \mathrm{com}-$ pares the survival curves of the pancreatic function in the subjects with the response in the lowest quartile $(<24.7$ $\mu \mathrm{U} / \mathrm{ml}$ ) to those with a higher response. The mean survival time was $4.2 \pm 0.76$ years in the subjects with a response in the lowest quartile, and $6.51 \pm 0.26$ years in the others $(P<0.0002$ with log-rank test). The bottom panel of Figure 3 shows the relationship between survival of the pancreatic function at 4 years and insulin response in the subjects who were transplanted at least 4 years previously. ROC analysis showed that a cutoff value of insulin delta peak $<32 \mu \mathrm{U} / \mathrm{ml}$ 
TABLE 2. Values derived from metabolic profiles and insulin clamp 1 year after the transplant

\begin{tabular}{|c|c|c|c|c|c|c|c|c|c|c|c|c|c|c|c|c|c|c|c|c|c|c|}
\hline \multirow{3}{*}{$\begin{array}{l}\text { subject } \\
\quad \text { id }\end{array}$} & \multicolumn{12}{|c|}{ 24-h profiles } & \multirow{3}{*}{$\begin{array}{c}\text { IVGTT } \\
\text { c-pep (ng/ml) } \\
\text { delta peak }\end{array}$} & \multirow{3}{*}{$\begin{array}{c}\text { Clamp } \\
\text { M value } \\
(\mathrm{mg} / \mathrm{kg} / \mathrm{min})\end{array}$} & \multicolumn{8}{|c|}{$\begin{array}{c}\text { Follow-up } \\
\text { yr }\end{array}$} \\
\hline & \multicolumn{4}{|c|}{ p-Glucose (mg/dL) } & \multicolumn{4}{|c|}{ f-iri $(\mu \mathrm{U} / \mathrm{ml})$} & \multicolumn{4}{|c|}{ c-pep (ng/ml) } & & & & & & & & & & \\
\hline & avg & $\min$ & $\max$ & delta & avg & $\min$ & $\max$ & delta & avg & $\min$ & $\max$ & delta & & & 1 & 2 & 3 & 4 & 5 & 6 & 7 & 8 \\
\hline 1 & - & - & - & - & - & - & - & - & - & - & - & - & 39.3 & 5.10 & 0 & 0 & 0 & 1 & 1 & 1 & 1 & 1 \\
\hline 2 & - & - & - & - & - & - & - & - & 4.1 & 2.1 & 9.0 & 6.9 & 20.6 & 5.22 & 0 & 0 & 0 & 0 & 0 & 0 & 1 & 1 \\
\hline 3 & - & - & - & - & - & - & - & - & - & - & - & - & -0.4 & 7.38 & 0 & 0 & 0 & 0 & 1 & 1 & 1 & 1 \\
\hline 4 & 137 & 84 & 276 & 192 & 35.3 & 10.8 & 81.5 & 70.7 & 5.4 & 3.0 & 9.6 & 6.6 & 31.3 & 6.10 & 0 & 1 & 1 & 1 & 1 & 1 & 1 & 1 \\
\hline 5 & 114 & 77 & 195 & 118 & 92.2 & 23.6 & 168.7 & 145.1 & 9.4 & 3.9 & 15.0 & 11.1 & 68.5 & 3.51 & 0 & 0 & 0 & 0 & 0 & 0 & - & - \\
\hline 6 & 108 & 84 & 139 & 55 & 26.1 & 18.0 & 52.8 & 34.8 & 3.3 & 2.0 & 5.9 & 3.9 & 60.4 & 3.93 & 0 & 0 & 0 & 0 & 0 & 0 & 0 & - \\
\hline 7 & 287 & 146 & 505 & 359 & 26.7 & 13.3 & 42.6 & 29.3 & 1.1 & 0.5 & 2.9 & 2.4 & 4.0 & 6.19 & 0 & 0 & 0 & 1 & 1 & 1 & 1 & 1 \\
\hline 8 & 110 & 74 & 161 & 87 & 19.4 & 5.8 & 41.1 & 35.3 & 3.1 & 1.4 & 6.3 & 4.9 & 49.3 & 6.75 & 0 & 0 & 0 & 0 & 0 & 0 & 0 & 0 \\
\hline 9 & 96 & 76 & 122 & 46 & 20.9 & 5.3 & 49.0 & 43.7 & - & - & - & - & 26.7 & 6.38 & 0 & 0 & 0 & 0 & 0 & 0 & 0 & 0 \\
\hline 10 & 117 & 98 & 153 & 55 & 30.7 & 14.3 & 50.9 & 36.6 & 6.1 & 3.7 & 9.9 & 6.2 & 30.2 & 4.93 & 0 & 0 & 0 & 0 & 1 & 1 & 1 & 1 \\
\hline 11 & 108 & 61 & 200 & 139 & 29.1 & 15.2 & 49.9 & 34.7 & - & - & - & - & & 5.56 & 0 & 0 & 0 & 0 & 1 & 1 & 1 & 1 \\
\hline 12 & 127 & 80 & 203 & 123 & 36.9 & 21.1 & 54.2 & 33.1 & - & - & - & - & 43.0 & - & 0 & 0 & 0 & 0 & 0 & 0 & 1 & 1 \\
\hline 13 & 141 & 89 & 243 & 154 & 20.3 & 5.5 & 45.3 & 39.8 & 3.6 & 2.1 & 6.3 & 4.2 & 22.9 & - & 0 & 1 & 1 & 1 & 1 & 1 & 1 & 1 \\
\hline 14 & 96 & 66 & 148 & 82 & 14.1 & 2.0 & 31.3 & 29.3 & 3.2 & 1.2 & 5.5 & 4.3 & 36.2 & - & 0 & 0 & 0 & 0 & - & - & - & - \\
\hline 15 & 163 & 114 & 222 & 108 & 37.0 & 11.2 & 83.2 & 72.0 & 3.7 & 2.2 & 5.3 & 3.1 & 7.2 & - & 1 & 1 & 1 & 1 & 1 & 1 & 1 & \\
\hline 16 & 134 & 92 & 261 & 169 & 26.0 & 13.5 & 48.1 & 34.6 & 5.2 & 2.7 & 8.5 & 5.8 & 13.1 & - & 0 & 0 & 0 & 0 & - & - & - & \\
\hline 17 & 104 & 85 & 130 & 45 & 35.8 & 12.6 & 72.5 & 59.9 & - & - & - & - & 132.1 & - & 0 & 0 & 0 & 0 & 0 & 0 & 0 & \\
\hline 18 & 130 & 84 & 246 & 162 & 39.8 & 15.7 & 153.3 & 137.6 & 5.9 & 2.9 & 16.8 & 13.9 & 43.0 & - & 0 & 0 & 0 & 1 & 1 & 1 & 1 & \\
\hline 19 & 125 & 98 & 210 & 112 & 54.0 & 12.0 & 164.7 & 152.7 & 7.9 & 4.2 & 14.1 & 9.9 & 90.9 & - & 0 & 0 & 0 & 0 & - & - & - & \\
\hline 20 & 90 & 74 & 113 & 39 & 16.9 & 5.9 & 41.2 & 35.3 & 5.9 & 3.6 & 8.8 & 5.2 & 71.9 & 3.31 & 0 & 0 & 0 & 0 & 0 & 0 & 0 & \\
\hline 21 & 99 & 87 & 139 & 52 & 19.1 & 6.2 & 88.7 & 82.5 & 6.5 & 4.0 & 12.4 & 8.4 & 70.4 & - & 0 & 0 & 0 & 0 & 0 & 0 & & \\
\hline 22 & 107 & 82 & 166 & 84 & 31.8 & 9.4 & 97.1 & 87.7 & 4.8 & 1.8 & 12.0 & 10.2 & 35.9 & 5.24 & 0 & 0 & 0 & 0 & 0 & 0 & & \\
\hline 23 & 111 & 84 & 155 & 71 & 44.1 & 18.3 & 108.0 & 89.7 & 4.3 & 2.6 & 7.7 & 5.1 & 75.9 & - & 0 & 0 & 0 & 0 & 0 & 0 & & \\
\hline 24 & 94 & 69 & 112 & 43 & 44.7 & 16.8 & 88.3 & 71.5 & 4.4 & 2.5 & 6.3 & 3.8 & 128.9 & 6.57 & 0 & 0 & 0 & 0 & 0 & 0 & & \\
\hline 25 & 103 & 82 & 182 & 100 & 37.1 & 14.3 & 75.6 & 61.3 & 5.0 & 2.3 & 9.4 & 7.1 & 68.2 & - & 0 & 0 & 0 & 0 & 0 & & & \\
\hline 26 & 97 & 65 & 151 & 86 & 70.2 & 2.8 & 192.1 & 189.3 & 12.1 & 4.6 & 23.0 & 18.4 & 123.1 & - & 0 & - & - & - & - & & & \\
\hline 27 & 105 & 83 & 138 & 55 & 42.6 & 14.1 & 92.4 & 78.3 & 4.9 & 2.3 & 8.0 & 5.7 & 132.5 & - & 0 & 0 & 0 & 0 & 0 & & & \\
\hline 28 & 176 & 127 & 242 & 115 & 26.5 & 17.9 & 42.3 & 24.4 & 2.2 & 1.6 & 3.0 & 1.4 & 0.3 & - & 1 & 1 & 1 & 1 & 1 & & & \\
\hline 29 & 109 & 88 & 162 & 74 & 43.1 & 17.6 & 92.4 & 74.8 & 4.4 & 2.3 & 8.0 & 5.7 & 84.8 & - & 0 & 0 & 0 & 0 & 0 & & & \\
\hline 30 & 92 & 66 & 122 & 56 & 17.3 & 7.3 & 32.6 & 25.3 & 2.5 & 1.8 & 3.9 & 2.1 & 107.0 & - & 0 & 0 & 0 & 0 & 0 & & & \\
\hline 31 & 112 & 79 & 190 & 111 & 45.5 & 15.7 & 104.6 & 88.9 & 7.1 & 2.3 & 12.7 & 10.4 & & - & 0 & 0 & 1 & 0 & 0 & & & \\
\hline 32 & 113 & 53 & 277 & 224 & 33.2 & 8.5 & 101.0 & 92.5 & - & - & - & - & 14.7 & - & 0 & 0 & 0 & 0 & & & & \\
\hline 33 & 106 & 84 & 154 & 70 & 48.9 & 18.1 & 120.8 & 102.7 & - & - & - & - & 209.3 & - & 0 & 0 & 0 & 0 & & & & \\
\hline 34 & 97 & 77 & 127 & 50 & 41.1 & 17.3 & 90.0 & 72.7 & 6.3 & 3.0 & 11.4 & 8.4 & 128.0 & - & 0 & 0 & 0 & 0 & & & & \\
\hline 35 & 132 & 84 & 212 & 128 & 46.5 & 12.9 & 123.3 & 110.4 & 5.1 & 2.4 & 8.0 & 5.6 & 12.4 & - & 0 & 0 & 0 & 1 & & & & \\
\hline 36 & 105 & 85 & 156 & 71 & 36.5 & 10.6 & 69.7 & 59.1 & 4.3 & 1.5 & 6.6 & 5.1 & 29.3 & - & 0 & 0 & 0 & 0 & & & & \\
\hline 37 & 129 & 82 & 247 & 165 & 27.4 & 10.3 & 70.1 & 59.8 & 3.5 & 1.6 & 6.3 & 4.7 & 40.7 & - & 0 & 0 & 0 & 0 & & & & \\
\hline 38 & 104 & 74 & 172 & 98 & 32.7 & 14.0 & 54.8 & 40.8 & 5.2 & 3.0 & 9.1 & 6.1 & 107.2 & - & 0 & 0 & 0 & 0 & & & & \\
\hline 39 & 101 & 74 & 148 & 74 & 60.8 & 14.3 & 190.2 & 175.9 & 5.7 & 2.9 & 11.3 & 8.4 & 97.4 & - & 0 & 0 & 0 & & & & & \\
\hline 40 & 116 & 80 & 197 & 117 & - & - & - & - & 5.5 & 3.2 & 10.3 & 7.1 & 50.4 & - & 0 & 0 & 0 & & & & & \\
\hline 41 & 138 & 102 & 235 & 133 & 26.7 & 11.2 & 44.3 & 33.1 & 4.3 & 2.2 & 6.8 & 4.6 & & - & 0 & 0 & 0 & & & & & \\
\hline 42 & 109 & 85 & 167 & 82 & 46.2 & 12.2 & 143.4 & 131.2 & 4.8 & 2.9 & 12.9 & 10.0 & 122.3 & - & 0 & 0 & 0 & & & & & \\
\hline 43 & - & - & - & - & 58.1 & 22.9 & 166.4 & 143.5 & 4.1 & 2.8 & 10.6 & 7.8 & 46.1 & - & 0 & 0 & 0 & & & & & \\
\hline 44 & 128 & 78 & 206 & 128 & 75.0 & 35.2 & 203.1 & 167.9 & 4.0 & 1.9 & 8.5 & 6.6 & 8.4 & - & 0 & 0 & 0 & & & & & \\
\hline 45 & 105 & 79 & 149 & 70 & 46.1 & 11.2 & 122.8 & 111.6 & 4.8 & 1.8 & 9.1 & 7.3 & & - & 0 & 0 & 0 & & & & & \\
\hline 46 & 119 & 84 & 230 & 146 & 17.4 & 2.1 & 76.0 & 73.9 & 5.2 & 2.1 & 13.1 & 11.0 & 53.3 & - & 0 & 0 & & & & & & \\
\hline 47 & 131 & 89 & 179 & 90 & 28.3 & 11.2 & 54.8 & 43.6 & 7.3 & 4.0 & 14.4 & 10.4 & 2.4 & - & 0 & 0 & & & & & & \\
\hline 48 & 99 & 77 & 141 & 64 & 15.2 & 3.1 & 26.9 & 23.8 & 3.2 & 1.3 & 4.9 & 3.6 & & - & 0 & 0 & & & & & & \\
\hline 49 & 114 & 73 & 159 & 86 & 31.8 & 10.1 & 71.2 & 61.1 & 9.9 & 4.5 & 18.4 & 13.9 & 67.6 & - & 0 & 0 & & & & & & \\
\hline 50 & 103 & 72 & 123 & 51 & - & - & - & - & - & - & - & - & 110.4 & - & 0 & 0 & & & & & & \\
\hline 51 & 112 & 68 & 161 & 93 & 22.0 & 2.8 & 69.4 & 66.6 & - & - & - & - & 8.7 & - & 0 & 0 & & & & & & \\
\hline 52 & 106 & 80 & 144 & 64 & 29.8 & 6.6 & 57.8 & 51.2 & - & - & - & - & 28.7 & - & 0 & 0 & & & & & & \\
\hline 53 & 122 & 87 & 158 & 71 & 103.1 & 22.6 & 250.6 & 228.0 & - & - & - & - & 199.7 & - & 0 & & & & & & & \\
\hline mean & 118 & 83 & 184 & 101 & 37.7 & 12.6 & 90.6 & 78.1 & 5.1 & 2.6 & 9.6 & 7.0 & 61.5 & 5.44 & & & & & & & & \\
\hline se & 4 & 2 & 9 & 8 & 2.7 & 0.9 & 7.5 & 7.1 & 0.3 & 0.1 & 0.7 & 0.5 & 7.3 & 0.33 & & & & & & & & \\
\hline $\mathrm{n}$ & 49 & 49 & 49 & 49 & 48 & 48 & 48 & 48 & 41 & 41 & 41 & 41 & 48 & 14 & & & & & & & & \\
\hline
\end{tabular}

The table reports the daily mean, minimum, maximum, and excursion of plasma glucose, serum free-insulin (firi), and c-peptide (c-pep) in the profiles, the insulin peak in the IVGTT, and the M value in the second hour of the euglycemic clamp performed 1 year after transplantation. A value 0 in the follow-up indicates free from diabetes; 1 , relapsed; and -, lost to follow-up for reasons independent from the pancreas. 


\section{METABOLIC PROFILES}
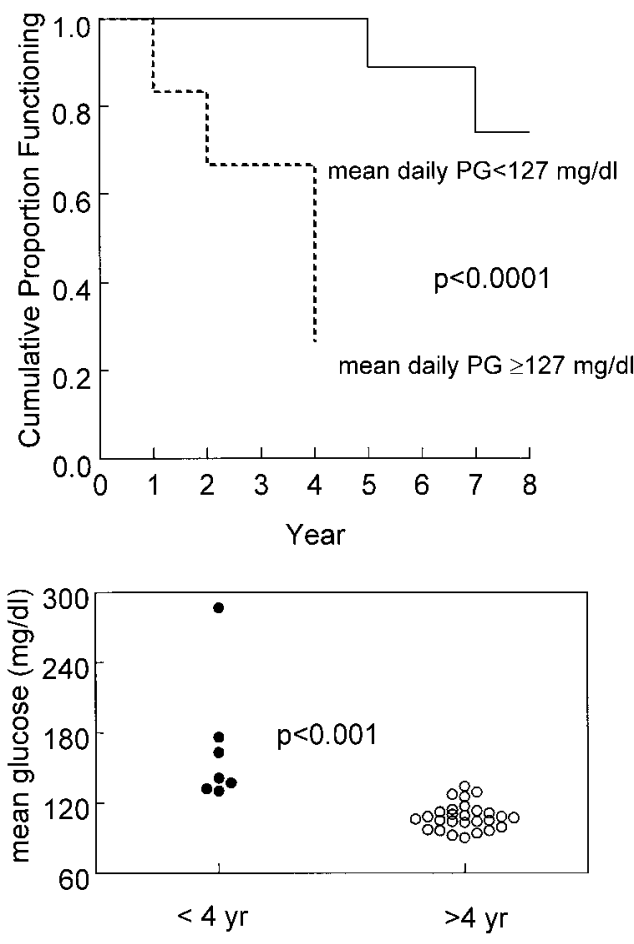

survival of pancreatic function

Figure 1. Top, An increased mean daily glucose in the profiles 1 year after transplantation was associated with a shorter duration of the pancreatic function. The figure shows the duration of pancreatic function in the subjects in the top quartile compared to the others. Bottom, The mean daily glucose was significantly increased in the subjects who lost the pancreatic function in the first 4 years.

predicts the return to the diabetic state within 4 years from transplantation with a $75 \%$ specificity and a $75 \%$ sensitivity.

\section{DISCUSSION}

This study showed that it is possible to predict with reasonable accuracy the return to the diabetic state for any cause in patients with type 1 diabetes who received a combined kidney and pancreas transplant. The best predictive index was the mean daily glucose concentration 1 year after transplantation, even though the minimum and the maximum daily glucose and the glycemic excursion had similar predictive powers. The subjects who could keep low their glucose concentration and could limit their daily glucose excursion had the best chances to remain free of diabetes after pancreas transplantation. The power of glucose profiles to predict failure in the long term may be surprising. Nonetheless, we also analyzed the predictive power of the metabolic profiles that the same subjects underwent 1 month after transplantation, and we achieved similar results (data not shown). These findings suggest that the reasons for pancreas failure are not contingent and the life span of the pancreas graft is at least in part already determined at the time of transplantation.

The ability to control glucose profiles depends both on insulin secretion and on insulin sensitivity. In regard to

\section{INSULIN CLAMP}
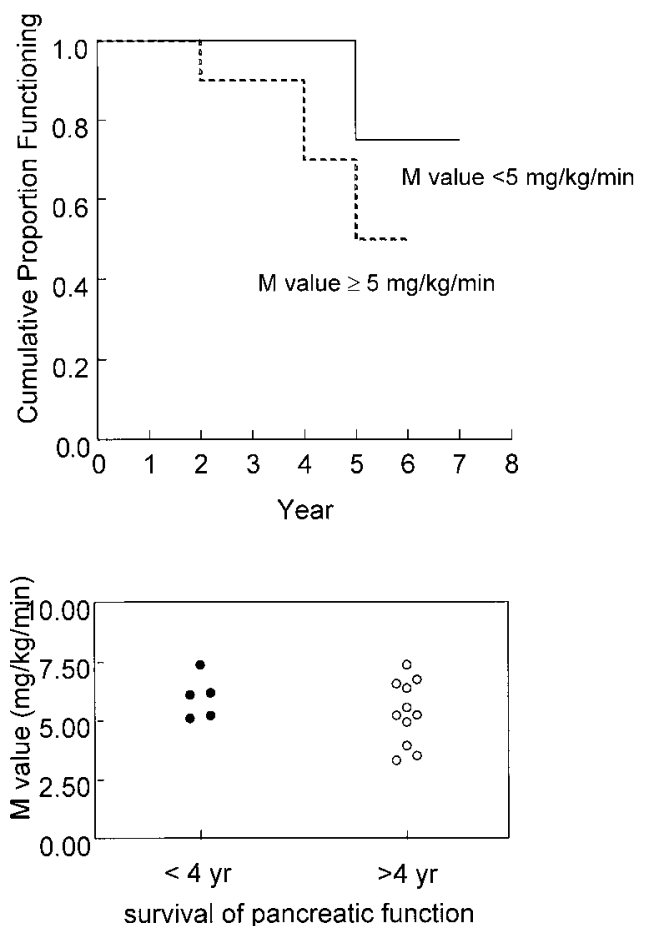

Figure 2. Top, A decreased M value in the clamp, an index of insulin sensitivity, 1 year after transplantation was not associated with the duration of the pancreatic function. The figure shows the duration of pancreatic function in the subjects in the lower quartile compared to the others. Bottom, The M value in the clamp was not decreased in the subjects who lost pancreatic function in the first 4 years.

insulin secretion, none of the indexes derived from the profiles of insulin and c-peptide was associated with the hazard function. The subjects with an earlier return to the diabetic state were not particularly insulinopenic or hyperinsulinemic, and they had insulinemic excursions comparable to the other subjects. The concentrations of the c-peptide showed a similar behavior. It is possible that the indexes derived from the insulin and the c-peptide daily profiles in this study lacked the sensitivity to detect defects in insulin secretion. In contrast, the IVGTT studies showed that a defective insulin response to intravenous glucose is associated with a return to the diabetic state. These data suggest that neither insulinopenia nor gross alterations in insulin secretion are significant early determinants of the return to the diabetic state. However, subtle defects such as the loss of the first phase of insulin secretion or altered insulin auto-feedback (16) may be involved in the early derangement of the glucose profiles and the subsequent return to the diabetic state.

There is no doubt that insulin sensitivity is another important factor in the derangement of glucose tolerance and the development of diabetes. Insulin resistance predicts the development of type 2 diabetes $(17,18)$, and it is also invariably associated with type 1 diabetes (19). We have previously shown that 1 year after transplantation, pancreas recipients are mildly insulin resistant for glucose metabolism (13). The posttransplant insulin resistance is at least in part caused by immunosuppression, and some predisposed subjects may be 


\section{IVGTT}


Figure 3. Top, A decreased peak insulin response to the IVGTT 1 year after transplantation was associated with a shorter duration of pancreatic function. The figure shows the duration of pancreatic function in the subjects in the lower quartile compared to the others. Bottom, The peak insulin response to the IVGTT was significantly decreased in the subjects who lost pancreatic function in the first 4 years.

particularly sensitive to the detrimental action of the steroids (20). Another reason for insulin resistance in heterotopic pancreas transplantation may relate to the peripheral site of insulin secretion (21). Nonetheless, insulin sensitivity in the recipients was devoid of predictive value for the return to the diabetic state. The small sample size $(n=14)$ limited the power for the statistical inference; however, the subjects who relapsed before 5 years from transplant had $M$ values well within 1 standard deviation of the distribution of the M values. Thus, it is unlikely that a larger sample could have shown that the $\mathrm{M}$ value is valuable for the long-term prediction of returning to the diabetic state.

Various reasons may explain why insulin resistance cannot predict the return to the diabetic state in the long term. Many factors that may affect the insulin sensitivity of recipients, including changes in the dose of the immunosuppressive drugs $(20)$, infections $(22,23)$, the renal function $(24)$, aging (25), and hyperinsulinemia (26), may change over time. We have previously shown that, as a consequence, the degree of insulin sensitivity changes over time after transplantation, presenting a steady improvement with fluctuations (13). Thus, insulin resistance cannot be considered per se an early risk factor, although it is an important last common pathway for many factors that determine the return to the diabetic state.

An additional explanation for the scarce value of measures of insulin sensitivity in predicting the long-term pancreatic function can be found in the close relationship that exists between insulin sensitivity and insulin secretion. Interestingly, in this study, the $\mathrm{M}$ value (an index of insulin sensitivity) was negatively associated with the mean concentration of c-peptide, which is an index of insulin secretion (Spearman rank-order correlation coefficient $=-0.6242$, $P=0.0480$ ). Insulin resistance and hyperinsulinemia are often associated. Insulin resistance, either primary (17) or secondary to steroid administration (27), can cause compensatory hyperinsulinemia. Conversely, hyperinsulinemia per $s e$, either by systemic diversion of the pancreatic drainage or by inappropriate insulin secretion, can cause insulin resistance $(21,26)$. In contrast, a decreased insulin secretion can initially increase insulin sensitivity $(28,29)$. However, in the long term, both insulin resistance and an increased and a decreased insulin secretion can equally lead to a decreased glucose tolerance. Thus, it is not surprising that neither insulin secretion nor insulin resistance were univocally associated to the mean glucose values in the profiles and to the risk of loosing the pancreatic function in the long term.

Irrespective of the causes of increased glucose concentrations in the recipients predisposed to pancreatic failure, our data suggest that hyperglycemia could adversely affect the outcome of the graft. The idea that chronic hyperglycemia per se impairs both insulin secretion and insulin sensitivity has been proposed several years ago (30), and it has been substantiated by a lot of experimental evidence (31-34). In the light of the present results, we propose to intensively monitor the subjects with a daily mean glucose concentration $>125$ $\mathrm{mg} / \mathrm{dL} 1$ year after transplantation, because these individuals have a high risk of returning to the diabetic state within 4 years. These are at-risk subjects who could benefit from aggressive programs for the early detection and treatment of pancreas rejection (2) and infection (35) and from the use of positive modulators of insulin action.

In conclusion, the return to the diabetic state is at least in part determined at the time of pancreas transplantation. Mean daily glucose concentrations are a good predictor of the return to the diabetic state, which is also associated to defects in insulin secretion and not to insulin resistance. Mean daily glucose concentrations 1 year after transplantation may be used to identify the recipients that could benefit from aggressive diagnostic and therapeutic protocols to prevent the recurrence of diabetes.

\section{REFERENCES}

1. Sutherland D, Gruessner A, Moudry-Munns K. International Pancreas Transplant Registry report. Transplant Proc 1994; 26: 407.

2. Klassen DK, Hoen-Saric EW, Weir MR, et al. Isolated pancreas rejection in combined kidney pancreas transplantation. Transplantation 1996; 61: 974.

3. Wong JJ, Krebs TL, Klassen DK, et al. Sonographic evaluation of acute pancreatic transplant rejection: morphology-Doppler analysis versus guided percutaneous biopsy. Am J Roentgenol 1996; 166: 803.

4. Jonsson P, Kallen R, Borgstrom A, Ohlsson K. Exocrine pancreatic proteins in serum during pancreatic allograft rejection. Pancreas 1994; 9 : 244.

5. Ploeg RJ, D'Alessandro AM, Groshek M, et al. Efficacy of human anodal trypsinogen for detection of rejection in clinical pancreas transplantation. Transplant Proc 1994; 26: 531.

6. Cheng SS, Munn SR. Posttransplant hyperamylasemia is associated with decreased patient and graft survival in pancreas allograft recipients. Transplant Proc 1994; 26: 428.

7. Abendroth D, Capalbo M, Illner WD, Landgraf R, Land W. Critical analysis of rejection markers sIL-2R, urinary amylase, and lipase in whole- 
organ pancreas transplantation with exocrine bladder drainage. Transplant Proc 1992; 24: 786.

8. Hartgrink HH, van Bockel JH, Hansen B, et al. Effect of blood group and HLA matching on pancreas graft survival with the use of UW solution. Transpl Int 1995; 8: 366.

9. Battezzati A, Bonfatti D, Benedini S, et al. Spontaneous hypoglycaemia after pancreas transplantation in type I diabetes mellitus. Diabet Med 1998; 15: 991.

10. Sollinger HW, Cook K, Kamps D, Glass NR, Belzer FO. Clinical and experimental experience with pancreaticocystostomy for exocrine pancreatic drainage in pancreas transplantation. Transplant Proc 1984; 16 : 749 .

11. Dubernard JM, Traeger J, Neyra P, Touraine JL, Tranchant D, BlancBrunat N. A new method of preparation of segmental pancreatic grafts for transplantation: trials in dogs and in man. Surgery 1978; 84: 633.

12. DeFronzo RA, Tobin JD, Andres R. Glucose clamp technique: A method for quantifying insulin secretion and resistance. Am J Physiol 1979; 237: E214.

13. Luzi L, Secchi A, Facchini F, et al. Reduction of insulin resistance by combined kidney-pancreas transplantation in Type 1 (insulin-dependent) diabetic patients. Diabetologia 1990; 33: 549.

14. Schmidt FH. Die enzimatische bestimmung von glucose und fructose nebeneinander. Klin Wschr 1961; 39: 1244.

15. Battezzati A, Luzi L, Perseghin G, et al. Persistence of counter-regulatory abnormalities in insulin-dependent diabetes mellitus after pancreas transplantation. Eur J Clin Invest 1994; 24: 751.

16. Luzi L, Battezzati A, Perseghin G, et al. Lack of feedback inhibition of insulin secretion in denervated human pancreas. Diabetes 1992; 41: 1632

17. DeFronzo RA. The triumvirate: $\beta$-cell, muscle, liver. A collusion responsible for NIDDM. Diabetes 1988; 37: 667.

18. Martin BC, Warram JH, Krolewski AS, Bergman RN, Soeldner JS, Kahn CR. Role of glucose and insulin resistance in development of type 2 diabetes mellitus: results of a 25-year follow-up study. Lancet 1992; 340: 925 .

19. DeFronzo RA, Hendler R, Simonson D. Insulin resistance is a prominent feature of insulin-dependent diabetes. Diabetes 1982; 31: 795.

20. Martinenghi S, Secchi A, Luzi L, Battezzati A, DiCarlo V, Pozza G. Acute deterioration of pancreatic graft function presumably determined by steroid-induced insulin resistance: a case report. Transplantation 1993; 56: 241.

21. Radziuk J, Barron P, Najm H, Davies J. The effect of systemic venous drainage of the pancreas on insulin sensitivity in dogs. J Clin Invest 1993; 92: 1713

22. Douglas RG, Shaw JHF. Metabolic response to sepsis and trauma. Br J
Surg 1989; 76: 115.

23. Frayn KN. Hormonal control of metabolism in trauma and sepsis. Clin Endocrinol (Oxf) 1986; 24: 577.

24. Castellino P, Solini A, Luzi L, et al. Glucose and amino acid metabolism in chronic renal failure: Effect of insulin and amino acids. Am J Physiol 1992; 262: F168.

25. Bonadonna RC, Groop LC, Simonson DC, DeFronzo RA. Free fatty acid and glucose metabolism in human aging: Evidence for operation of the Randle cycle. Am J Physiol 1994; 266: E501.

26. Battezzati A, Terruzzi I, Perseghin G, et al. Defective insulin action on protein and glucose metabolism during chronic hyperinsulinemia in subjects with benign insulinoma. Diabetes 1995; 44: 837.

27. Rizza RA, Mandarino LJ, Gerich JE. Cortisol-induced insulin resistance in man: Impaired suppression of glucose production and stimulation of glucose utilization due to a postreceptor detect of insulin action. J Clin Endocrinol Metab 1982; 54: 131.

28. Barron P, Goobi P, Davies J, Pye S, Radziuk J. Hemipancreatectomy, peripheral diversion of pancreatic venous drainage, and insulin sensitivity. Transplant Proc 1995; 27: 3040.

29. Kergoat M, Guerre-Millo M, Lauva M, Portha B. Increased insulin action in rats with mild insulin deficiency induced by neonatal streptozotocin. Am J Physiol 1991; 260: E561.

30. Unger RH, Grundy S. Hyperglycaemia as an inducer as well as a consequence of impaired islet cell function and insulin resistance: implications for the management of diabetes. Diabetologia 1985; 28: 119.

31. Yki-Järvinen H, Helve E, Koivisto VA. Hyperglycemia decreases glucose uptake in type I diabetes. Diabetes 1987; 36: 892.

32. Del Prato S, Leonetti F, Simonson DC, Sheehan P, Matsuda M, DeFronzo RA. Effect of sustained physiologic hyperinsulinaemia and hyperglycaemia on insulin secretion and insulin sensitivity in man. Diabetologia 1994; 37: 1025.

33. Boden G, Ruiz J, Kim CJ, Chen X. Effects of prolonged glucose infusion on insulin secretion, clearance, and action in normal subjects. Am J Physiol 1996; 270: E251.

34. Robertson RP, Olson LK, Zhang HJ. Differentiating glucose toxicity from glucose desensitization: a new message from the insulin gene. Diabetes 1994; 43: 1085.

35. Lumbreras C, Fernandez I, Velosa J, Munn S, Sterioff S, Paya CV. Infectious complications following pancreatic transplantation: incidence, microbiological and clinical characteristics, and outcome. Clin Infect Dis 1995; 20: 514 .

Received 27 December 1999.

Accepted 12 September 2000. 